\title{
Article \\ Stochastic Gravity and Ontological Quantum Mechanics
}

\author{
Thomas C Andersen 1 (iD) \\ 1 NSCIR - 8 Bruce St, B 841, Thornbury, Ontario, Canada; tandersen@nscir.ca
}

\begin{abstract}
Some physicists surmise that gravity lies outside of quantum mechanics. Thus theories like the standard semiclassical theory of quantum to gravity coupling (that of Rosenfeld and Møller) are possible real models of interaction, rather than a mere approximation of a theory of quantum gravity. Unfortunately, semiclassical gravity creates inconsistencies such as superluminal communication. Alternatives by authors such as Diósi, Martin, Penrose, and Wang often use the term 'stochastic' to set themselves apart from the standard semiclassical theory. These theories couple to fluctuations caused by for instance continuous spontaneous localization, hence the term 'stochastic'. This paper looks at stochastic gravity in the framework of a class of emergent or ontological quantum theories, such as those by Bohm, Cetto, and de Broglie. It is found that much or all of the trouble in connecting gravity with a microscopic system falls away, as Einstein's general relativity is free to react directly with the microscopic beables. The resulting continuous gravitational wave radiation by atomic and nuclear systems does not, in contrast to Einstein's speculation, cause catastrophic problems. The small amount of energy exchanged by gravitational waves may have measurable experimental consequences. A very recent experiment by Vinante et al. performed on a small cantilever at $\mathrm{mK}$ temperatures shows a surprising non-thermal noise component, the magnitude of which is consistent with the stochastic gravity coupling explored here.
\end{abstract}

Keywords: Quantum Gravity; Emergent Quantum Mechanics; Gravitational Waves, General Relativity

\section{From semiclassical to stochastic gravity}

Semiclassical gravity can be summarized as a classical gravitational field coupled to quantum matter fields. While semiclassical gravity is widely thought of as a workable limiting approximation until a quantum theory of gravity is discovered, there are researchers who treat semiclassical gravity as a real possibility and hence in need of experimental tests[1]. The semiclassical equations for quantum gravity are as from Møller[2] and Rosenfeld[3]:

$$
R_{\mu \nu}-\frac{1}{2} g_{\mu \nu} R=\frac{8 \pi G}{c^{4}}\left\langle\Psi\left|T_{\mu v}\right| \Psi\right\rangle
$$

While seemingly straightforward, semiclassical gravity has problems, especially in determining the quantum expectation value (see Appendix A of Bahrami[4]). Superluminal signalling is also an issue[5].

To address these issues, Tilloy and Diósi[5], Martin[6], Penrose[7] and Wang[8] invoke mechanisms which solve some of the problems of semiclassical gravity. Diósi-Penrose state reduction, for example asserts that[7]:

...an expectation that quantum superpositions of states involving a significant mass displacement should have a finite lifetime...

Wang, Zhu and Unruh[8] describe a hugely fluctuating metric of space time down to scales at and below the Planck length. 
The key difference from the usual semiclassical gravity is that we go one more step-instead of assuming the semiclassical Einstein equation, where the curvature of the spacetime is sourced by the expectation value of the quantum field stress energy tensor, we also take the huge fluctuations of the stress energy tensor into account. In our method, the sources of gravity are stochastic classical fields whose stochastic properties are determined by their quantum fluctuations.

In these theories the gravitational field is stochastically coupled to matter. In other words the gravitational field fluctuates much more than in the original semiclassical formalism.

\section{Stochastic Gravity in EmQM}

Stochastic gravity in ontological quantum mechanics can be simple. Einstein's general relativity can couple directly to the sub-quantum 'beables' in these theories. If one assumes an emergent quantum mechanics delivered by a mechanism such as SEDs[9][10] or other theories such as[11][12][13][14][15][16], then perhaps Einstein's equations hold without modification down to at least the Planck scale. This would on some scale defy quantum mechanics as some stationary states would for instance radiate gravitational waves continuously. Violating quantum mechanics is usually not a good things for a theory, but as many dynamical collapse models[17][5] do, we assume that the required changes to quantum theory will be small.

What are experimental consequences of such an supposition? Start by looking at the 1916 Bohr planetary atom.

\subsection{The (non) collapse of the Bohr atom}

Ashtekar[18] cites Einstein in 1916:

...Nevertheless, due to the inner-atomic movement of electrons, atoms would have to radiate not only electro-magnetic but also gravitational energy, if only in tiny amounts. As this is hardly true in Nature, it appears that quantum theory would have to modify not only Maxwellian electrodynamics, but also the new theory of gravitation.

While his prediction for electromagnetism was prescient, perhaps Einstein's 'hardly true in Nature' quip was ill considered. Consider the energy loss rate of a circa 1916 style Bohr planetary hydrogen atom in the ground state, using Eddington's[19] formula for the gravitational energy radiated by a two body system (in the approximation that one mass is much heavier):

$$
d E / d t(\text { atom })=-\frac{32 G m_{e}^{2} r_{h}^{4} \omega^{6}}{5 c^{5}}=-10^{-43} \mathrm{eV} / \mathrm{s}
$$

Which even over the age of the universe amounts to an energy loss due to gravitational waves for a hydrogen atom in the ground state of only $10^{-25} \mathrm{eV}$. Why was Einstein worried about such a small rate of gravitational energy loss for a hydrogen atom? In contrast the electromagnetic lifetime of the classical hydrogen atom is about $10^{-11} \mathrm{~S}$ which of course helped lead to the discovery of quantum mechanics.

This energy loss is of no experimental significance. So we can conclude that the stability of atomic orbitals is not an experimental indication of a need for quantum gravity. In other words we cannot experimentally determine if atoms radiate gravitational waves continuously or not. Of course quantum mechanics demands that atoms do not radiate anything in their ground states, no matter what their angular momentum!

\subsection{Gravitational radiation from within nuclei}

The nucleus has much heavier and more densely packed particles than the electronic orbitals of atomic physics. With the nucleons being 2000 time the mass of an electron, and only femtometres apart, the possibility for gravitational wave emission is much higher. 
The motion of the individual nuclei is an accepted fact in the world of experimental nuclear physics. For instance, the nuclear physics book Nuclear Dynamics In The Nucleonic Regime[20] states

As already suggested, independent particle motion is a key feature of nuclei in their ground state.

If we assume that this motion is directly felt by classical general relativity, we can estimate the flux of gravitational waves emitted from a nucleus. To get a feel for the numbers involved, we first look at nuclear generated gravitational waves in an astrophysical context.

Weinberg, in his 1972 book[21] calculates the thermal gravitational wave emission of the Sun to be about $79 \mathrm{MW}$ at atomic frequencies. In a similar manner, Sivram - Arun[22] calculate the gravitational wave emission from other bodies such as neutron stars. Sivaram - Arun's calculations imply a gravitational wave emission rate of $10^{-16} \mathrm{eV} / \mathrm{s}$ per neutron[22], using their neutron star calculation. Neutron stars are in some respects like 'huge nuclei', with temperatures and densities of roughly that of an atomic nucleus. Therefore we can use $10^{-16} \mathrm{eV} / \mathrm{s}$ as an estimate of the emission rate for atomic nuclei, assuming that gravitational waves are emitted by the motions of nucleons.

Gravitational wave fluxes near the Sivaram - Arun levels hint that such effects might be measurable in the lab. If an atomic nuclei emit and/or absorb on the order of $10^{-16} \mathrm{eV} / \mathrm{s}$ (perhaps even per nucleon), then a nucleus might exchange about $\sim 1000 \mathrm{eV}$ over the lifetime of the universe.

\section{Experimental consequences}

This continuous gravitational wave emission is very small in comparison to the normal energy exchange caused by thermal noise that a nucleus suffers in normal matter. Nevertheless adding continuous stochastic gravitational wave physics into nuclear and sold state physics might bring some experimental consequences. With the above estimates of per nucleon gravitational wave emission, a $\mathrm{kg}$ of matter might be radiating (and perhaps absorbing)

$$
d E / d t(k g \text { of matter }) \sim 10^{-16} \frac{\mathrm{eV}}{\text { nucleon s }} 1^{26} \text { nucleons } \sim 10 \mathrm{GeV} / \mathrm{s}
$$

Experiments similar to those done to look for 'big G' might be a place where an as yet unmeasured energy exchange might alter the results. It's notable that experiments to determine Newton's constant $G$ have had great difficulty obtaining consistent results. Most measurements of $G$ do not agree with each other to within the errors carefully determined by the experimenters[23].

\subsection{A current experiment}

Experiments minimizing and measuring thermal noise using cryogenic techniques are a good candidate to see these effects. The recent experiment by Vinante et al. detailed in Improved noninterferometric test of collapse models using ultracold cantilevers[24] finds:

The finite intercept, clearly visible in the inset of Fig. 3 implies that the data are not compatible with a pure thermal noise behavior, and a nonthermal excess noise is present. 


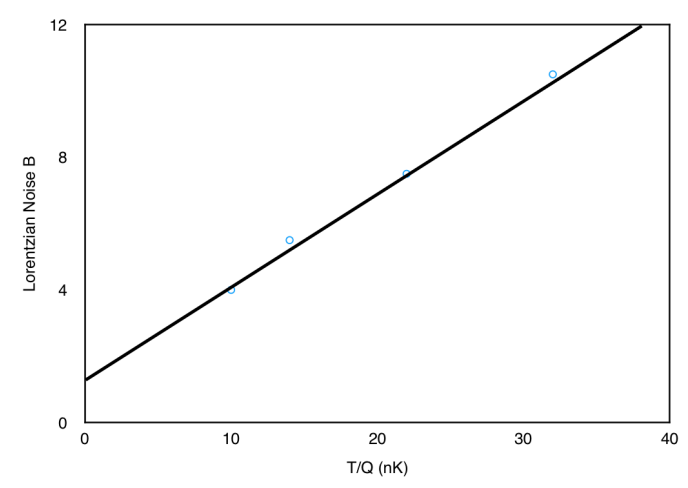

Figure 1. Data from figure 3 Vinante[24] - T/Q is the ratio of temperature to the Quality value of the cantilever. If noise was solely thermal the fit would intercept at the graph origin. The lowest temperature data point $(\mathrm{T} / \mathrm{Q}=10 \mathrm{nK})$ is $43 \mathrm{mK}$, so from the graph, the the excess nonthermal noise is as if the cantilever has an excess noise in the tens of $\mathrm{mK}$ range.

Could this noise be the result of gravitational wave generation or absorption? There are about $3 \times 10^{15}$ atoms of silicon in the arm, which is $0.5 \mathrm{~mm}$ long and $0.05 \mathrm{~mm}$ wide. Given the above emission/absorption rate of about $1 \times 10^{-16} \mathrm{eV} / \mathrm{sec}$ per atom, we can use the Stefan-Boltzmann law to get a temperature equivalent of the energy flowing in the system - which works out to $77 \mathrm{mK}$.

$$
T^{4}=\frac{\text { EnergyFlow }}{\text { Area } \sigma}=\frac{\left(1 \times 10^{-16} \mathrm{eV} / \mathrm{s}\right)\left(3 \times 10^{15} \text { atoms }\right)}{\text { Area } \sigma} \Longrightarrow T \sim 0.077 \mathrm{~K}
$$

This is of the order of the excess noise measured in the experiment. This can be seen by for instance looking at the lowest data point in 1, or by using Vinante's $Q$ value of $\sim 10^{7}$ at $20 \mathrm{mK}$.

\section{Emission/Absorption Parameter Space}

If gravitational waves are being absorbed and emitted by nucleons (and/or quarks), a central question is the amplitude of the stochastic gravitational wave background. This amplitude is directly related to the cross section for absorption of these particles. Calculating a cross section for something like a pair of nucleons is possible if one assumes that the nucleons behave like amorphous blobs of nuclear material. This calculation though only provides a lower limit to a cross section. The cross sections would be much higher if the microstructure of the nucleons was optimized to 'catch' gravitational waves.

An analogous story: If one were to estimate the cross section of a wind turbine knowing nothing but the mass of the turbine (say 64 tonnes) and construction material (steel), the calculated cross section would be tiny, as a physicist would model the 'wind turbine' as some sort of 3 metre in diameter solid ball of steel, with the ability to extract just about exactly no energy from the wind. By arranging that steel into an efficient bladed aerofoil (with $2 \%$ lightweight material added), the cross section of the now reasonably designed wind turbine is millions of times higher.

Similarly to the story about wind turbines above, if gravitational waves are involved in nuclear physics at all, it may be that nature has optimized the cross section of nuclei to gravitational waves, rather than minimized it.

A conceptual graph showing the large unknown parameter space of cross section vs emission rate is shown in FIG. 2. 


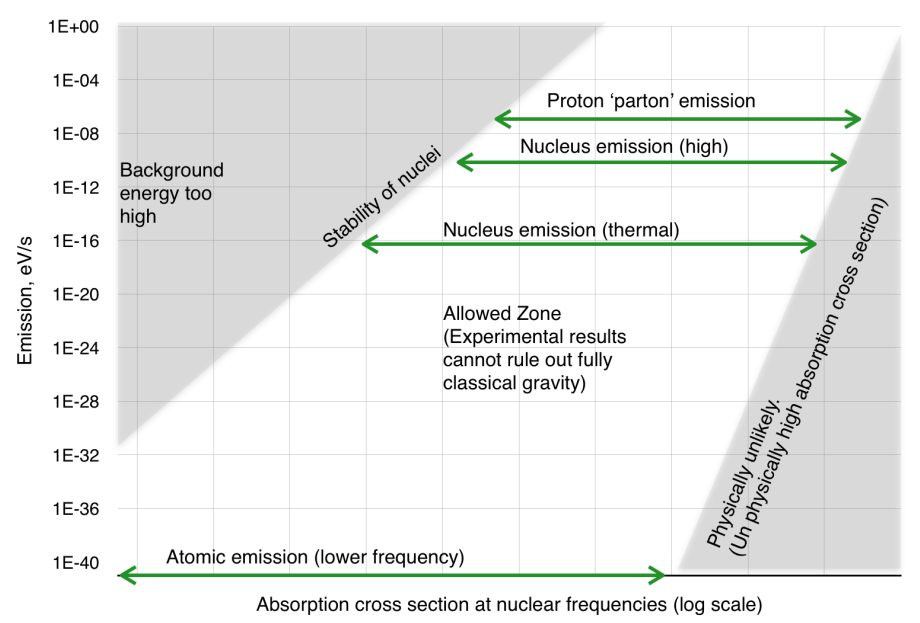

Figure 2. Nuclear frequency gravitational wave emission and absorption. The elusive nature of gravitational wave detection means that even fully classical quantum gravity cannot be experimentally ruled out. The frequency of the gravitational waves is that of nucleons ( $\omega \approx 10^{22} \mathrm{~Hz}$ ).

FIG. 2 is a sketch of allowed emission and absorption parameters. Some - but not all - combinations of emission and absorption parameters are ruled out by experiment. Towards the upper left of the image limited absorption combined with higher emission would mean that the stochastic background of gravitational waves would be so energetic as to have already been seen, or would interfere with normal quantum behavoir. The phrase 'stability of nuclei' refers to the experimental fact that nuclei live for billions of years. On the right a ruled out region exists where absorption cross sections are not physically likely. The top line shows a calculation for the gravitational wave flux of a proton due to parton (quark) motion. 'Nuclear emission (high)' refers to the Eddington flux for a heavy nucleus, while the lower nucleus flux is calculated assuming thermal Coulomb gravitational wave emission inside each nucleus.

\section{Discussion}

Due to the weak nature of gravitational effects on subatomic particles, even fully classical gravity cannot be experimentally ruled out at this time. Perhaps experiments such as Vinante's[24] will turn into a new tool to explore the interface of quantum mechanics and gravity.

These tests are also a test of the ubiquity of quantum mechanics. With a quantum violating result the conceptual foundations of quantum mechanics would be in question, as gravity would then be determined to be outside of the realm of quantum mechanics.

Acknowledgments: The author appreciates the travel and conference grant by the organizers of EMQM 2017, where the author presented a poster titled 'Stochastic Gravity and Ontological Quantum Mechanics'. The author also thanks the Fetzer Franklin Fund for assistance with the costs to publish in this open access journal.

Conflicts of Interest: The author declares no conflict of interest.

\section{References}

1. Großardt, A. Newtonian self-gravity in trapped quantum systems and experimental tests. arXiv:1702.04309 [quant-ph].

2. Møller, C. Les theories relativistes de la gravitation. Colloques Internationaux CNRS, Paris 1962, 91.

3. ROSENFELD, L. ON QUANTIZATION OF FIELDS. Physics, Nuclear Co, North-holland Publishing 1963, pp. 353-356.

4. Bahrami, M.; Großardt, A.; Donadi, S.; Bassi, A. The Schrödinger-Newton equation and its foundations. arXiv:1407.4370 [quant-ph] 2014. 
5. Tilloy, A.; Diósi, L. Sourcing semiclassical gravity from spontaneously localized quantum matter 2015. [1509.08705]. doi:10.1103/PhysRevD.93.024026.

6. Martin, R.; Verdaguer, E. Stochastic semiclassical gravity 1999. [arXiv:gr-qc/9904021]. doi:10.1103/PhysRevD.60.084008.

7. Penrose, R. On the Gravitization of Quantum Mechanics 1: Quantum State Reduction. Found Phys 2014, 44, 557-575. doi:10.1007/s10701-013-9770-0.

8. Wang, Q.; Zhu, Z.; Unruh, W.G. How the huge energy of quantum vacuum gravitates to drive the slow accelerating expansion of the Universe. Physical Review D 2017, 95, 103504. doi:10.1103/PhysRevD.95.103504.

9. de la Peña, L.; Cetto, A.M.; Valdés Hernández, A. The Emerging Quantum; 2015; p. 371. doi:10.1007/978-3-319-07893-9.

10. Cetto, A.M.; de la Peña, L. Real vacuum fluctuations and virtual Unruh radiation. Fortschritte der Physik 2016, 7, 1-7. doi:10.1002/prop.201600039.

11. Andersen, T.C. Can a sub-quantum medium be provided by General Relativity? Journal of Physics: Conference Series 2016, 701, 012023. doi:10.1088/1742-6596/701/1/012023.

12. Grössing, G.; Fussy, S.; Mesa Pascasio, J.; Schwabl, H. Implications of a deeper level explanation of the deBroglie-Bohm version of quantum mechanics. Quantum Studies: Mathematics and Foundations 2015, 2, 133-140, [1412.8349]. doi:10.1007/s40509-015-0031-0.

13. Brady, R.; Anderson, R. Why bouncing droplets are a pretty good model of quantum mechanics 2014. [1401.4356].

14. Bush, J.W. Pilot-Wave Hydrodynamics. Annual Review of Fluid Mechanics 2015, 47, $269-292$. doi:10.1146/annurev-fluid-010814-014506.

15. van Holten, T. The Atomic World Spooky? It ain't neccesarily so!; 2015.

16. Nieuwenhuizen, T.M. Towards Einstein's dream of a unified field theory: Reports from a journey on a long and winding road. Journal of Physics: Conference Series 2012, 361, 012036. doi:10.1088/1742-6596/361/1/012036.

17. Adler, S.L.; Bassi, A. Quantum Theory: Exact or Approximate? 2009. [0912.2211].

18. Ashtekar, A.; Reuter, M.; Rovelli, C. From General Relativity to Quantum Gravity. arXiv:1408.4336 [gr-qc] 2014, [1408.4336].

19. Eddington, A.S. The Propagation of Gravitational Waves. Proceedings of the Royal Society of London A: Mathematical, Physical and Engineering Sciences 1922, 102, 268-282.

20. Durand, D.; Suraud, E.; Tamain, B. NUCLEAR DYNAMICS IN THE NUCLEONIC REGIME; IOP Publishing, 2001; p. 522. doi:https://doi.org/10.1201/9781420033793.

21. Weinberg, S. Gravitation and cosmology : principles and applications of the general theory of relativity; Wiley, 1972; p. 657.

22. Sivaram, C. Thermal Gravitational Waves. arXiv:0708.3343 2007, [0708.3343]. doi:10.2174/1874381101004010065.

23. Rosi, G.; Sorrentino, F.; Cacciapuoti, L.; Prevedelli, M.; Tino, G.M. Precision measurement of the Newtonian gravitational constant using cold atoms. Nature 2014, 510, 518-21, [1412.7954]. doi:10.1038/nature13433.

24. Vinante, A.; Mezzena, R.; Falferi, P.; Carlesso, M.; Bassi, A. Improved noninterferometric test of collapse models using ultracold cantilevers 2016. [1611.09776]. 